\title{
SCHLUCKFUNKTION NACH LASERCHIRURGISCHER THERAPIE VON OROPHARYNXKARZINOMEN
}

6

\section{F. Bosse*, B. Bestmann+, Th. Küchler+, St. Maune*}

${ }^{*}$ Klinik für Hals-, Nasen-, Ohrenheilkunde, Kopf- und Halschirurgie Universitätsklinikum S-H, Campus Kiel (Direktorin: Prof. Dr. P. Ambrosch) +Interdisziplinäres Referenzzentrum für Lebensqualität in der Onkologie, Universitätsklinikum S-H, Campus Kiel (Leiter: Priv. Doz. Dr. Th. Küchler)

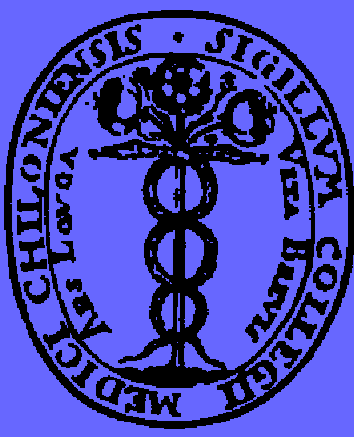

A. D. 1665

Zusammenfassung:: Die Laserchirurgie bei Oropharynxkarzinomen wurde in den in den letzten Jahren zu einem etablierten Therapieansatz entwickelt. Die Schluckfunktion variiert dabei in Abhängigkeit von Art und Umfang der Therapie. Analysiert wird der zeitliche Verlauf der Einschränkung der Schluckfunktion als ein Standardendpunkt zur Beurteilung des postoperativen Ergebnisses.

\section{Einleitung:}

Im Rahmen umfassender Vorarbeiten wurde in Zusammenarbeit mit dem Referenzzentrum für LQ in der Onkologie an der CAU zu Kiel ein Konzept zur Erfassung der LQ von Patienten mit Karzinomen des HNO Fachgebietes entwickelt. Als Grundlage eines Gesamtfragebogens wurde der CoreFragebogen der European Organisation for Reasearch and Treatment of Cancer (EORTC), der EORTC C-30 ausgewählt. Ergänzt wurde dieser durch ein fachspezifisches Modul, den EORTC H\&N 35, ein in Kiel entworfenes therapiespezifisches Modul KQL H\&N 17 und einen sozio-ökonomischen Fragebogen.

Dieser Gesamtfragebogen wird seit März 2000 an alle Patienten mit einem Karzinom des oberen Luft- Speisetraktes verteilt. Die Erfassung erfolgt sowohl prä- als auch in regelmäßigen Abständen posttherapeutisch. Zusätzlich werden alle medizinischen Daten der Patienten numerisch kodiert und so einer statistischen Auswertung zugänglich gemacht.

\section{Patienten und Methode:}

Täglich werden die Stationen unserer Klinik auf Neuaufnahmen mit V.a. Erstdiagnose eines PECA des oberen Aerodigestivtraktes kontrolliert. So ist eine lückenlose Erfassung der Patienten gesichert. Im Rahmen eines Aufklärungsgespräches werden die Patienten dann über Sinn und Ablauf der Studie informiert. Anschließend erhalten die Patienten, ihr Einverständnis vorausgesetzt, den ersten Fragebogen. Kann die Einwilligung des Patienten trotz Aufklärungsgespräch nicht eingeholt werden, so wird der Grund des Ausfalles dokumentiert.

Posttherapeutisch werden die erfassten Patienten in regelmäßigen Abständen in die Nachsorgesprechstunde einbestellt und erhalten zusätzlich zur medizinischen Nachsorge eine Befragung ihrer empfundenen LQ.

Die erhobenen Daten werden anschließend numerisch kodiert und sind so einer statistischen Auswertung z.B. mit SPSS zugängig.

\section{Ergebnisse:}

Dargestellt werden die Ergebnisse von 67 Patienten mit Laserresektionen von Oropharynxkarzinomen.

\section{Symptomskala "Dysphagie" des EORTC H\&N 35}

Die subjektiv empfundene Qualität wird dargestellt auf einer Skala von 0-100. Ein höherer Wert entspricht einer schlechteren Funktion. Zur Ermittlung der Werte werden die Ergebnisse von 4 Einzelfragen des Moduls zusammengefasst.

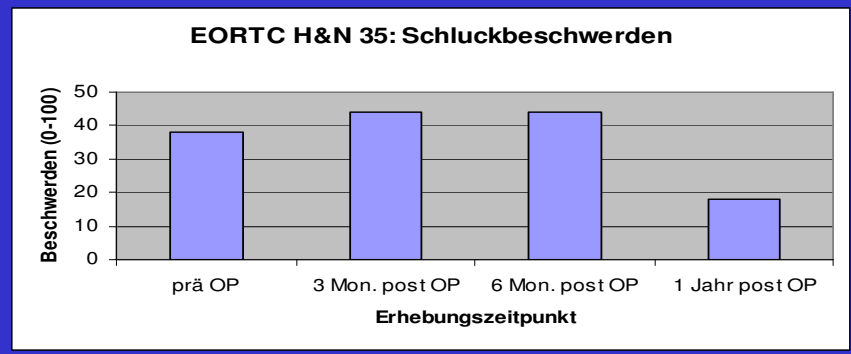

Entwicklung der Schluckfunktion bei Patienten mit einem Oropharynx CA im zeitlichen Verlauf
Schluckfunktion nach Nahrungskonsistenz. Dargestellt wird die Schluckfunktion in Abhängigkeit von der Konsistenz der Nahrung auf einer Skala von 0-100 im Vergleich „prätherapeutisch“ zu "1 Jahr posttherapeutisch“. (0=keine Beschwerden).

Schmerzen beim Schlucken: Dargestellt werden die Schmerzen beim Schluckakt in gleicher Skalierung im Vergleich „prätherapeutisch“ zu "1 Jahr posttherapeutisch". (0=keine Beschwerden).

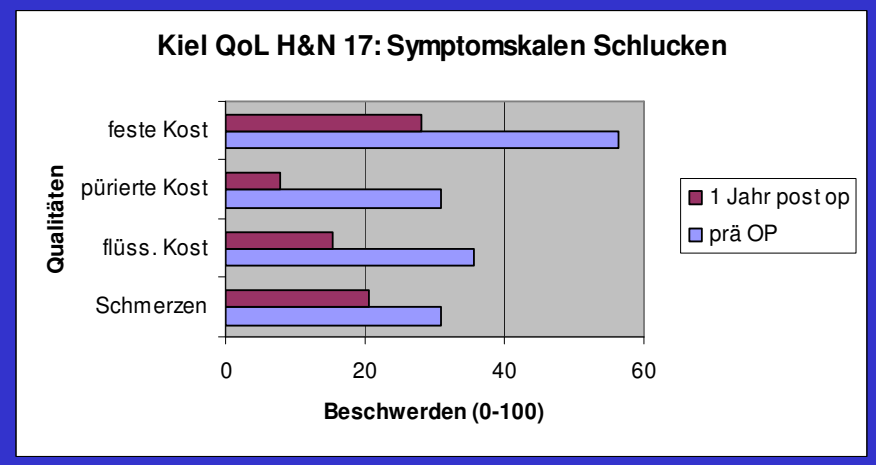

Entwicklung der Schluckfunktion nach Nahrungskonsistenz und der Schmerzen beim Schluckakt bei Patienten mit einem Oropharynx CA, unterteilt nach zeitlichem Verlauf

\section{Diskussion:}

Die Entwicklung der Schluckfunktion bei Patienten mit Laserresektion eines Oropharynxkarzinoms zeigt einen Verlauf mit persistierenden Beschwerden über ein halbes Jahr. Erst nach Ablauf eines Jahres wird von den Patienten eine verbesserte Schluckfunktion angegeben, die dann im Vergleich zum prätherapeutischen Zustand klinisch signifikant besser ist.

Bestätigt werden diese Ergebnisse durch Einzelitems aus dem KQL H\&N 17. Dabei zeigt sich für die Schluckfunktion unterschiedlicher Nahrungskonsistenzen jeweils eine klinisch signifikante Verbesserung über den Zeitraum von einem Jahr. Resultierende Schlussfolgerungen:

1.Durch die laserchirurgische Behandlung von Oropharynxkarzinomen ist eine deutliche Verbesserung der Schluckfunktion und der Schluckschmerzen zum prätherapeutischen Status zu erwarten.

2. Die Aufklärung der Patienten über die Operation sollte eine Information über den im Vergleich zur normalen Wundheilung deutlich verlängerten Prozess der Normalisierung des Schluckaktes beinhalten.

3. Aufgrund der Tatsache, dass auch ein Jahr posttherapeutisch die Nahrungsaufnahme fester Speisen die größten Beschwerden bereitet, sollten logopädische Therapiemaßnahmen einen festen Platz in der postoperativen Rehabilitation einnehmen.

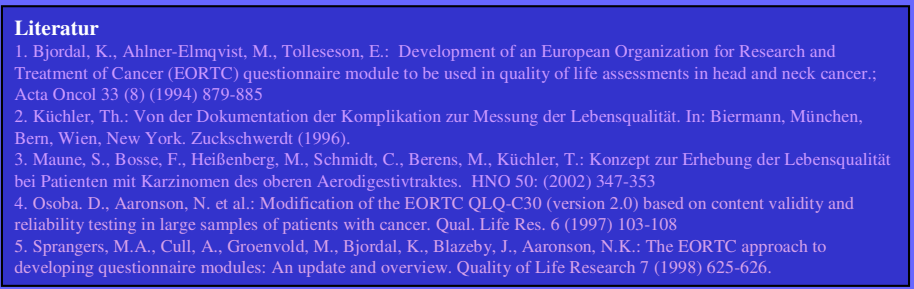

